\title{
Deep Groundwater Recharge Mechanism in the Sedimentary and Crystalline Terrains of Sri Lanka: A Study Based on Environmental Isotopic and Chemical Signatures of Spring Water
}

\author{
Sakhila Priyadarshanee ${ }^{1}$, Zhonghe Pang ${ }^{2}$, Viraj Edirisinghe ${ }^{3}$, H.A. Dharmagunawardhane ${ }^{4}$, \\ H.M.T.G.A. Pitawala ${ }^{4}$, J.D.C. Gunasekara ${ }^{3}$, and I.A.N.P.D. Tilakarathne ${ }^{5}$ \\ ${ }^{1}$ Institute of Geology and Geophysics Chinese Academy of Sciences \\ ${ }^{2}$ Institute of Geology and Geophysics \\ ${ }^{3}$ Sri Lanka Atomic Energy Board \\ ${ }^{4}$ University of Peradeniya \\ ${ }^{5}$ National Water Supply and Drainage Board
}

November 27, 2020

\begin{abstract}
In many instances, dynamic, potential status and geochemical characteristics of groundwater discharging through natural springs are not well known. Present study has assessed the deep groundwater in the form of thermal and non-thermal spring in artesian condition in the selected zones in Sri Lanka, using isotope and geochemical characteristics. The results revealed that evaporation-fractional crystallization and cation-exchange in the sedimentary aquifers while rock-water interaction in crystalline deep aquifers, are the significant mechanism that control the groundwater chemistry. All the deep groundwater recharged from meteoric water at different elevations and further influenced by either evaporation or rock-water interaction during the subsurface flow. Artesian aquifers in the sedimentary terrain in the north-western coastal zones showed the recharging elevation as from 100 to $200 \mathrm{~m}$ amsl. They are not mixed with sea water and slightly impacted by the locally evaporated surface waters. Almost all these waters are comparatively old; indicating slow movement along the regional flow paths. Considering the recharge and discharge conditions of artesian non-thermal waters in the Southern lowlands of crystalline terrain can be classified as non-mixed, non-evaporated and young groundwater with higher elevation recharge. The artesian non-thermal waters in the East North Central lowlands, have shown the same characteristics but with evaporated conditions. All artesian thermal waters are tritium free, hence they are older and deep percolated. Intensive rock-water interaction and higher altitude origin were observed in some thermal springs. Some spring clusters in the weathered overburden have shown significant mixing with recent local rains. Non-mixed, non-evaporated and less rock-water interacted nature is a significant in two thermal springs that emerges through (chemically inert) quartzite bed rock. Both thermal and non-thermal water with artesian condition have clearly indicated that they are originated from a common recharge source but with different flow paths in different penetration depths and travel distances, resulting different chemical characteristics. Fresh water springs are mostly young and recharged from local rains followed with shallow percolation.
\end{abstract}

\section{Hosted file}

Main Document.pdf available at https://authorea.com/users/379359/articles/495588-deepgroundwater-recharge-mechanism-in-the-sedimentary-and-crystalline-terrains-of-sri-lankaa-study-based-on-environmental-isotopic-and-chemical-signatures-of-spring-water 

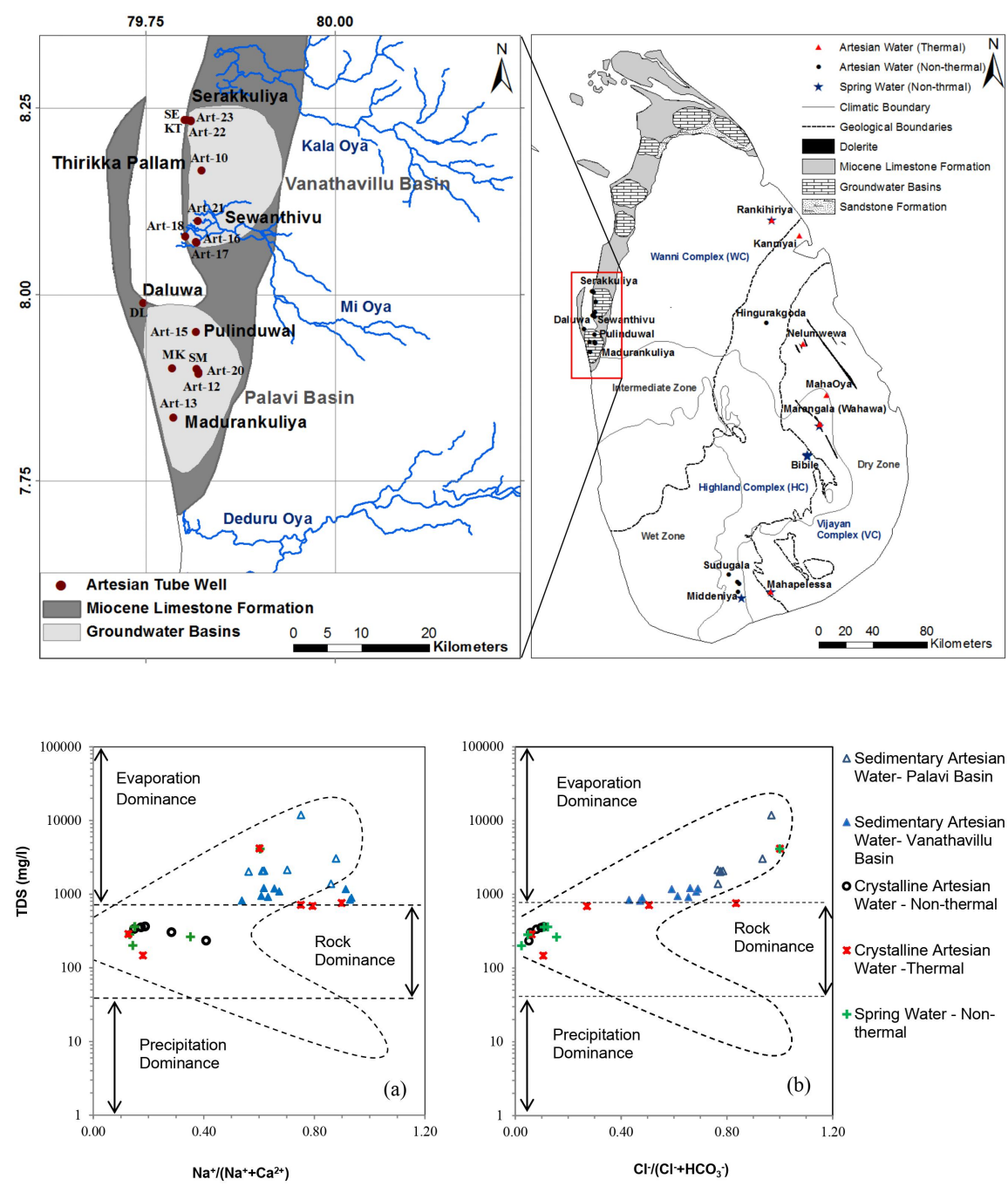

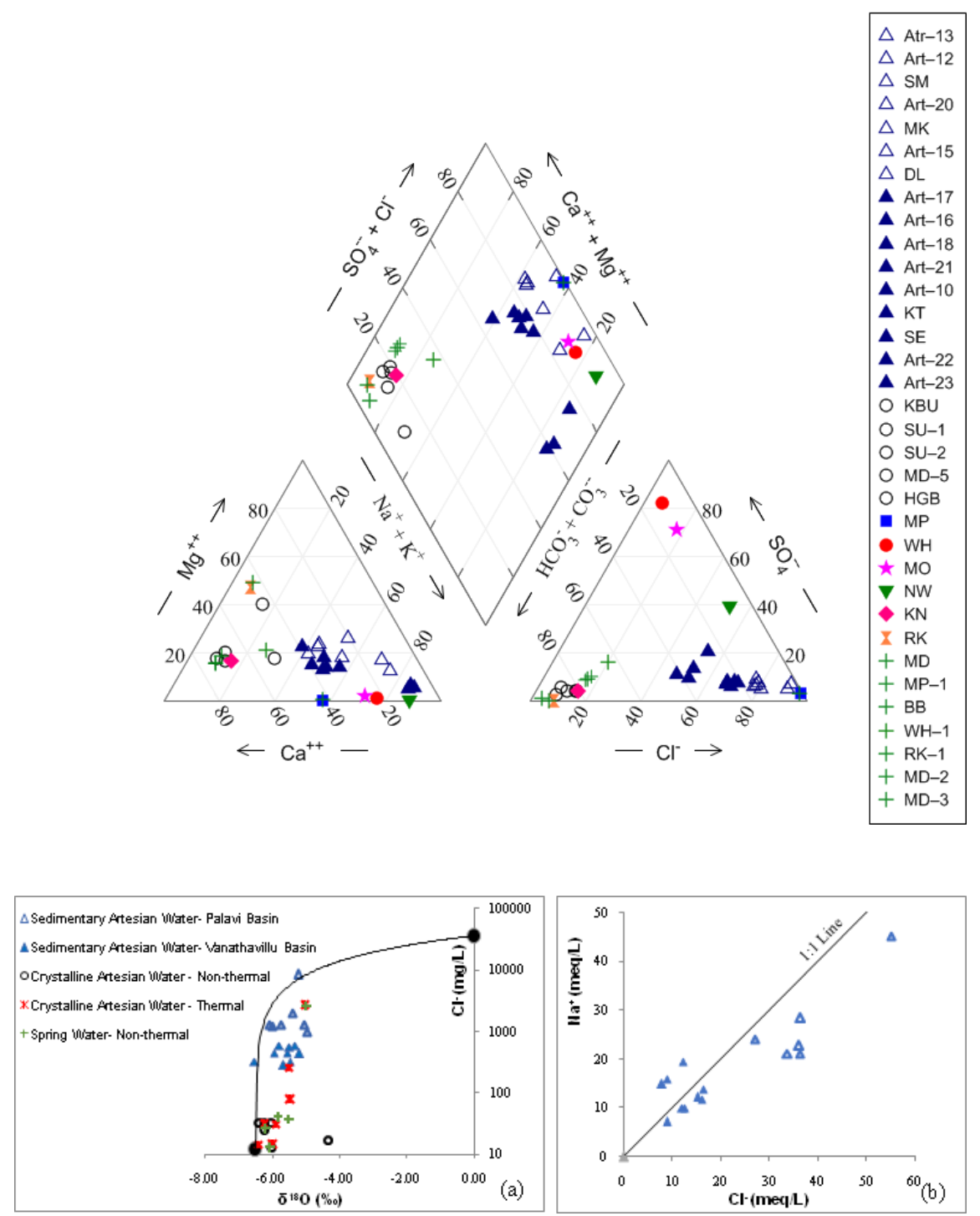

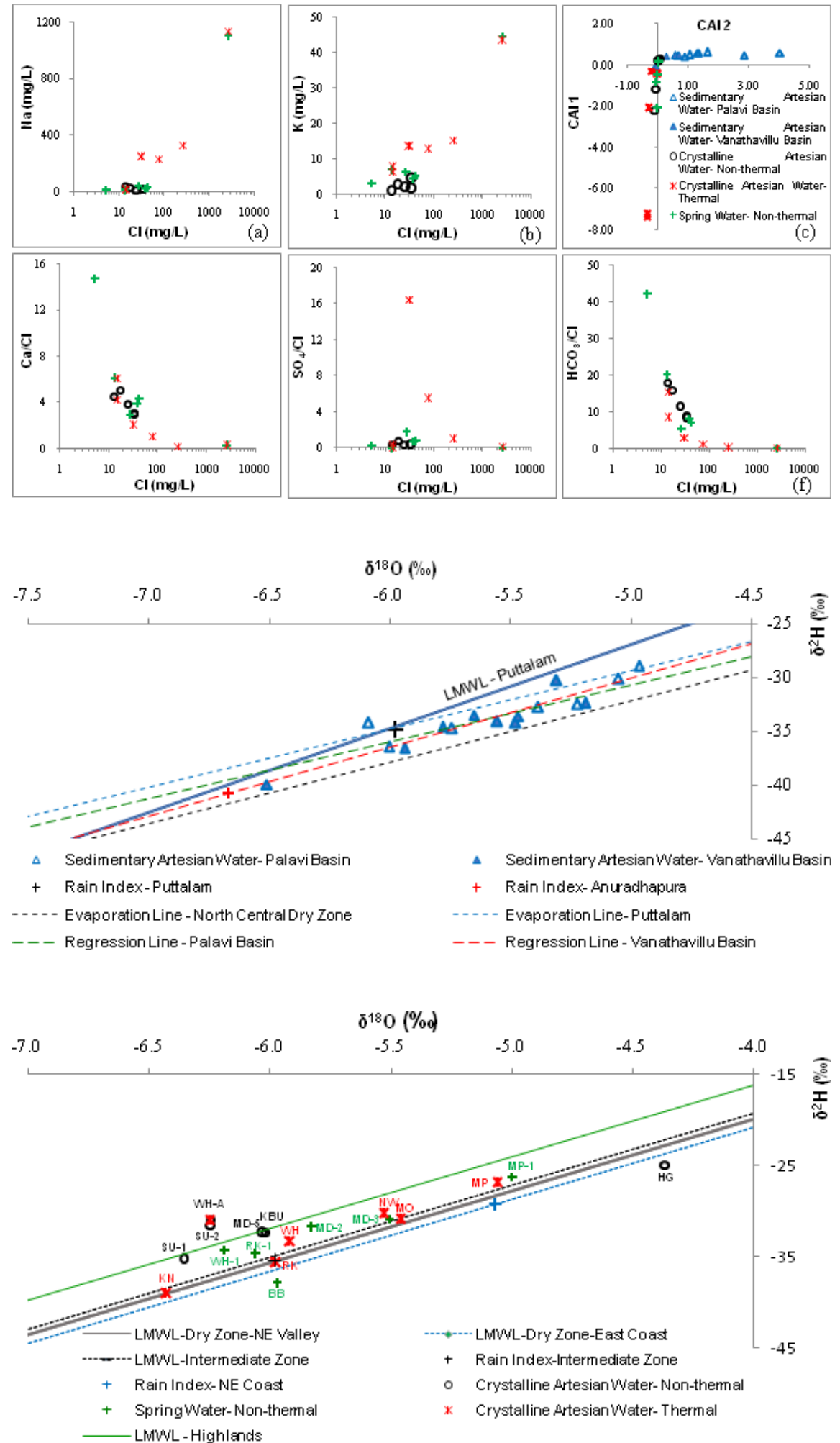

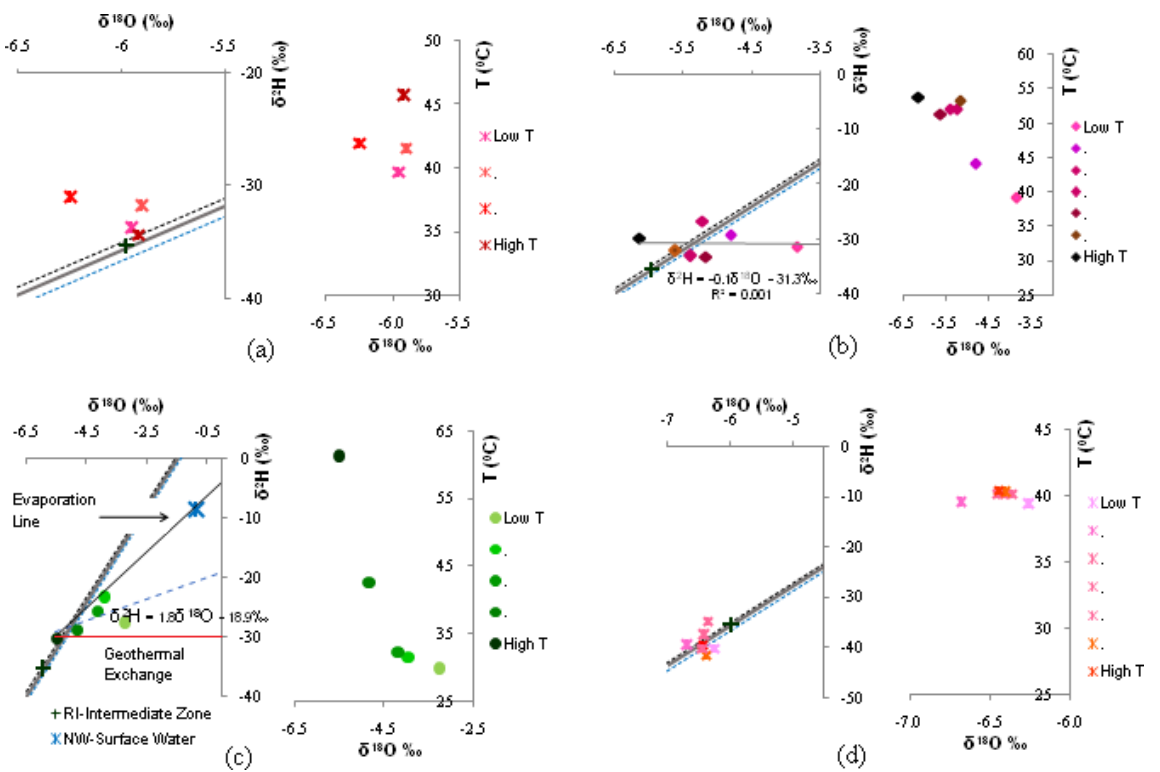

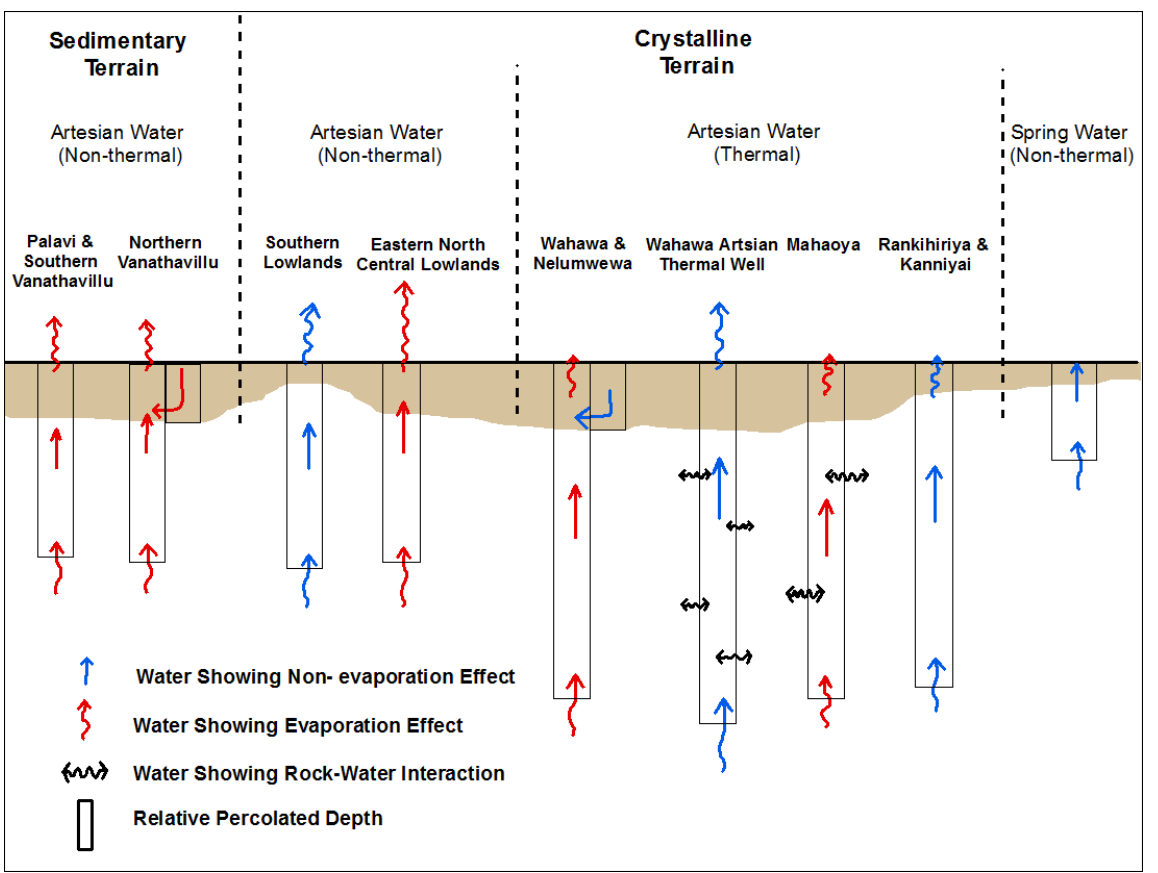




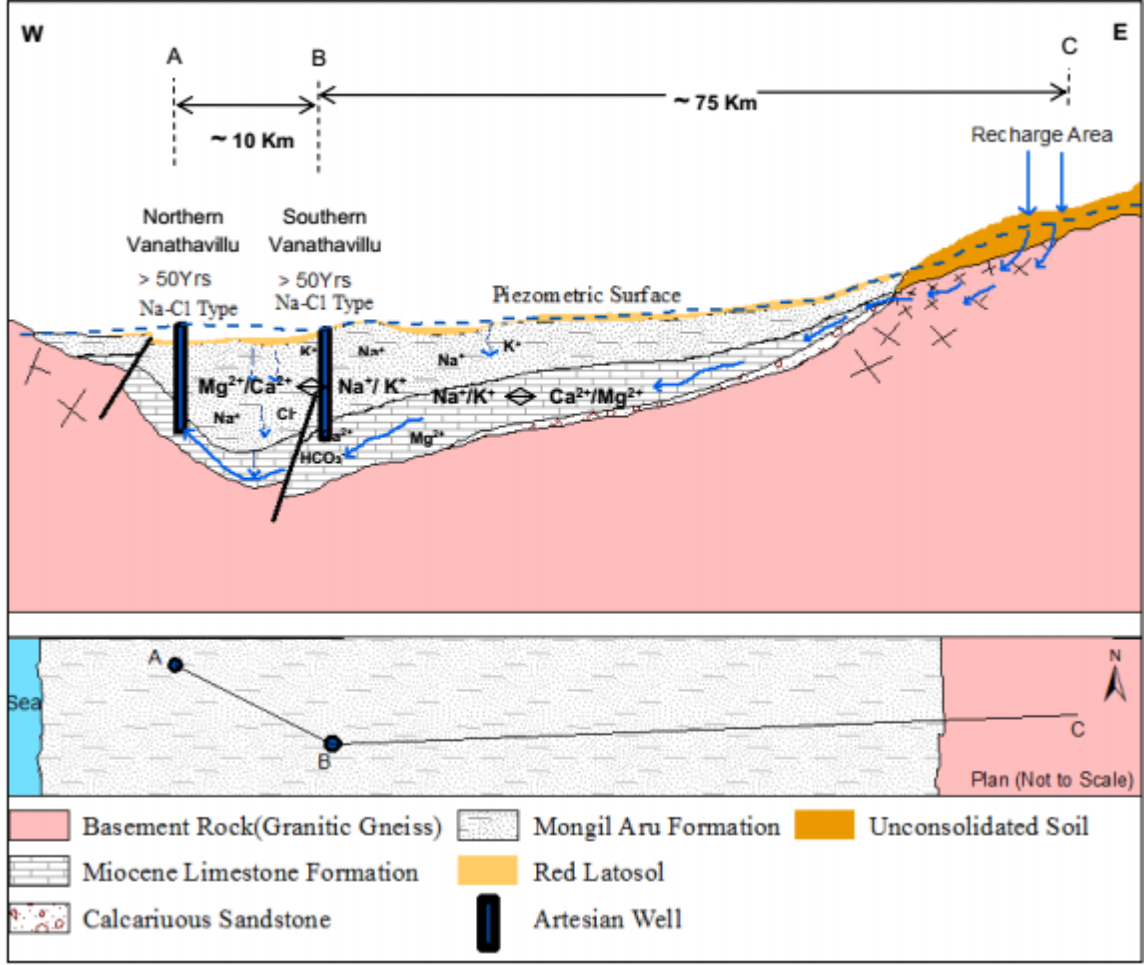




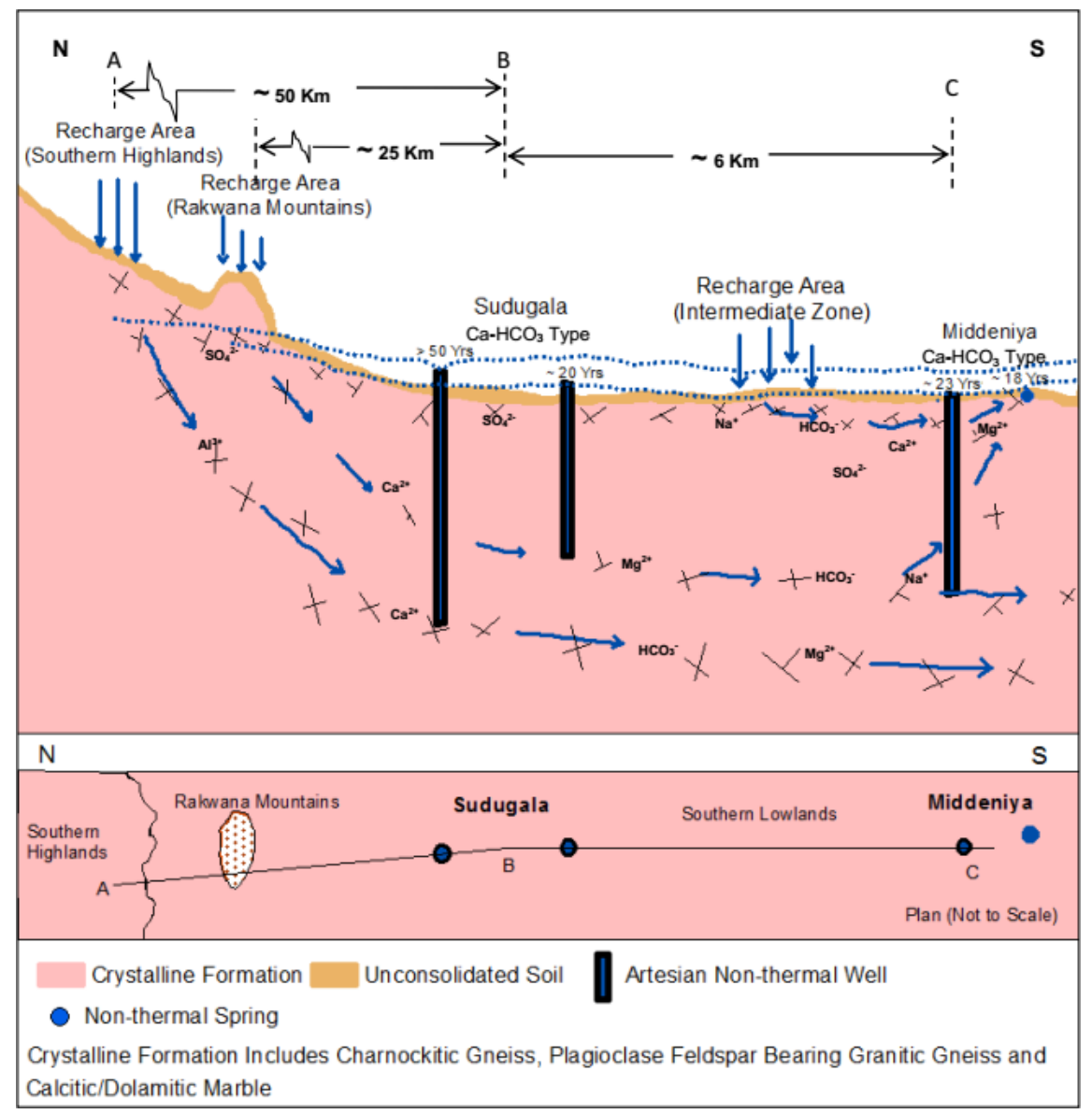




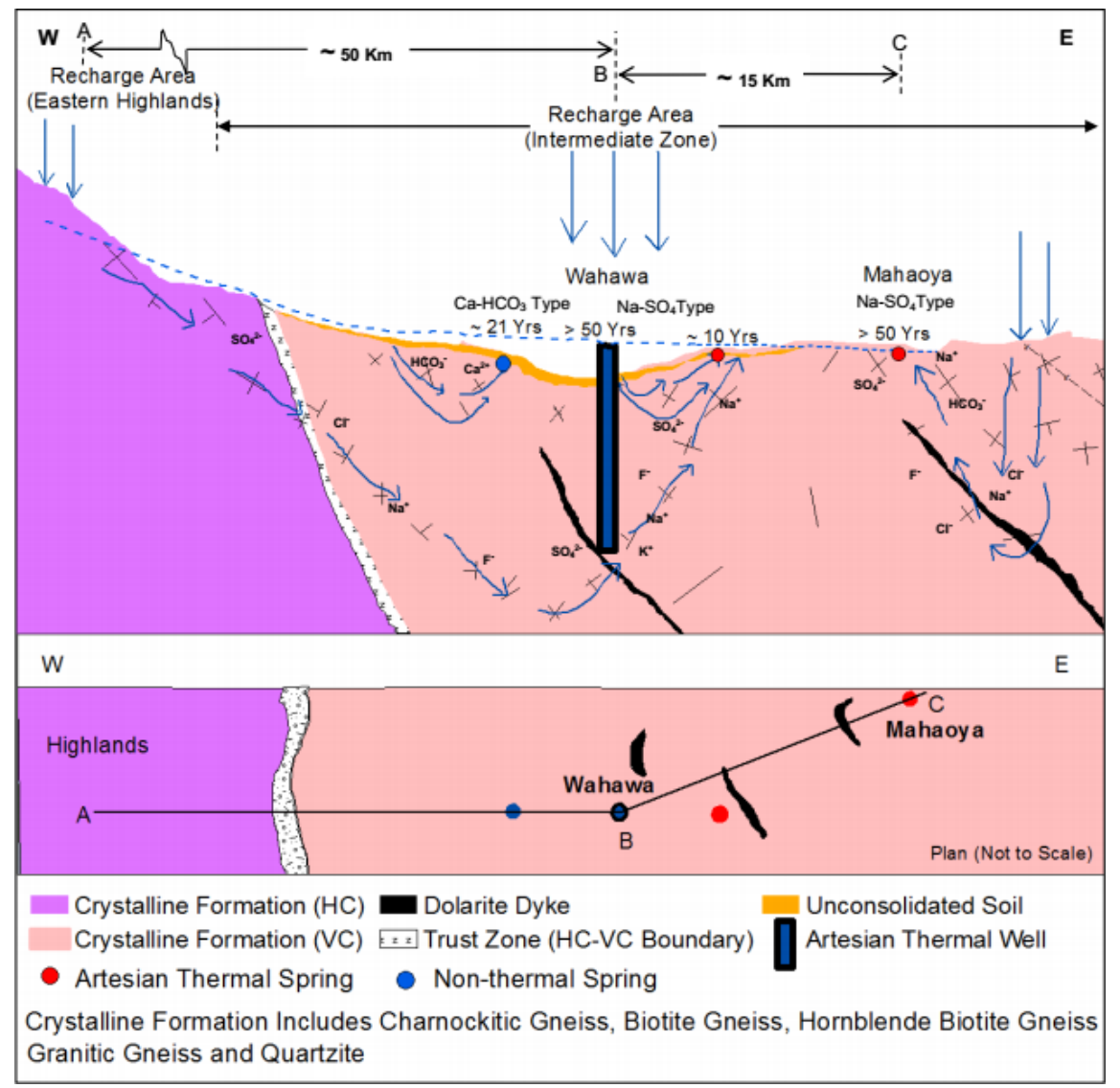




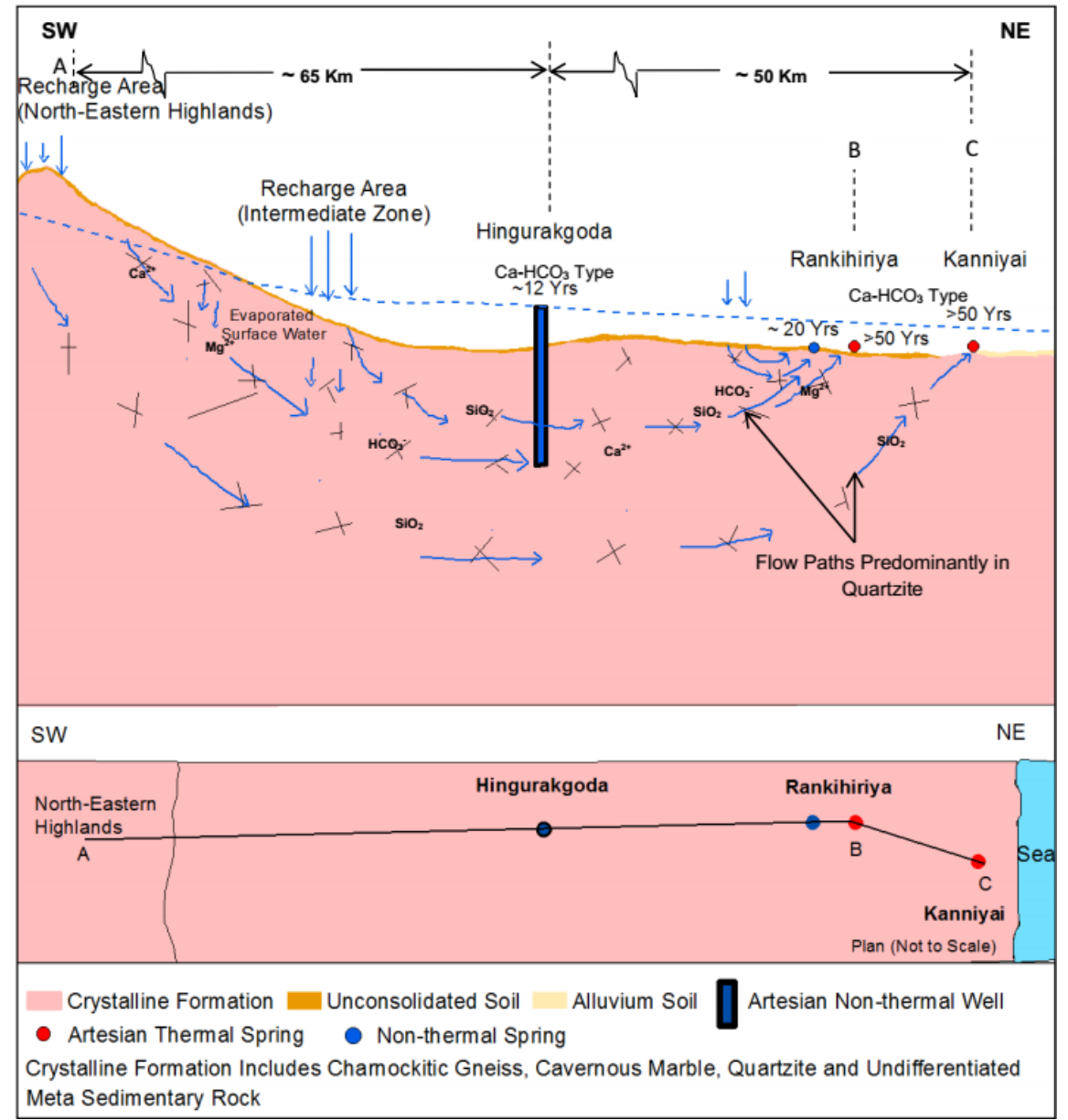

\section{Hosted file}

TABLE 1.pdf available at https://authorea.com/users/379359/articles/495588-deep-groundwaterrecharge-mechanism-in-the-sedimentary-and-crystalline-terrains-of-sri-lanka-a-studybased-on-environmental-isotopic-and-chemical-signatures-of-spring-water 
TABLE 2: Rain indexes, LMWLs and evaporation lines of different locations and climatic zones

\begin{tabular}{|c|c|c|c|c|}
\hline \multirow{2}{*}{$\begin{array}{l}\text { Rain Collecting } \\
\text { Location }\end{array}$} & \multicolumn{2}{|c|}{ Rain Index (RI) } & \multirow{2}{*}{$\begin{array}{c}\text { Local Meteoric Water Line (LMWL)/ } \\
\text { Evaporation Line }\end{array}$} & \multirow[t]{2}{*}{ References } \\
\hline & $\delta^{18} \mathbf{O}(\%)$ & $\delta^{2} \mathrm{H}(\%)$ & & \\
\hline Puttalam & -5.98 & -34.8 & $\begin{array}{l}\delta^{2} \mathrm{H}=7.8 \delta^{18} \mathrm{O}+12.1 \%\left(\mathrm{r}^{2}=0.97\right) \\
\text { Evaporation Line: } \delta^{2} \mathrm{H}=5.4 \delta^{18} \mathrm{O}-2.3 \%\end{array}$ & Edirisinghe et al., 2014 \\
\hline Anuradhapura & -6.67 & -40.7 & $\begin{array}{l}\delta^{2} \mathrm{H}=7.9 \delta^{18} \mathrm{O}+12.2 \%\left(\mathrm{r}^{2}=0.94\right) \\
\text { Evaporation Line }- \text { North Central DZ: } \\
\delta^{2} \mathrm{H}=5.7 \delta^{18} \mathrm{O}-3.6 \%\end{array}$ & Edirisinghe et al., 2017 \\
\hline Highland $(\sim 1400 \mathrm{~m})$ & & & $\delta^{2} \mathrm{H}=7.9 \delta^{18} \mathrm{O}+15.4 \%$ & Measured data in 2016 \\
\hline Intermediate Zone & -5.98 & -35.3 & $\delta^{2} \mathrm{H}=7.9 \delta^{18} \mathrm{O}+12.4 \%$ & Edirisinghe et al., 2017 \\
\hline Dry Zone - East Cost & & & $\delta^{2} \mathrm{H}=7.9 \delta^{18} \mathrm{O}+10.8 \%$ & Edirisinghe et al., 2017 \\
\hline Dry Zone - NE Valley & & & $\delta^{2} \mathrm{H}=7.9 \delta^{18} \mathrm{O}+11.8 \%$ & Edirisinghe et al., 2017 \\
\hline
\end{tabular}




\begin{tabular}{|c|c|c|c|c|c|}
\hline Water Group & & $\begin{array}{l}\text { Recharge } \\
\text { Zone }\end{array}$ & $\begin{array}{l}\text { Discharging Water } \\
\text { Condition }\end{array}$ & $\begin{array}{l}\text { MRT } \\
\text { (Yrs) }\end{array}$ & $\begin{array}{l}\text { Water } \\
\text { Type }\end{array}$ \\
\hline \multirow[t]{2}{*}{$\begin{array}{l}\text { Artesian Water in the } \\
\text { Sedimentary Terrain }\end{array}$} & $\begin{array}{l}\text { (a).Palavi and } \\
\text { Southern part of } \\
\text { Vanathavillu basin }\end{array}$ & $\begin{array}{l}100 \text { to } 200 \mathrm{~m} \\
\text { altitude }\end{array}$ & $\begin{array}{l}\text { Insignificantly mixed with } \\
\text { surface water, slightly } \\
\text { evaporated }\end{array}$ & $>50$ & $\mathrm{Na}-\mathrm{Cl}$ \\
\hline & $\begin{array}{l}\text { (b). Northern part of } \\
\text { Vanathavillu Basin }\end{array}$ & $\begin{array}{l}100 \text { to } 200 \mathrm{~m} \\
\text { altitude }\end{array}$ & $\begin{array}{l}\text { Slightly mixed with percolated } \\
\text { water through Mongil Aru } \\
\text { Formation, slightly evaporated }\end{array}$ & $>50$ & $\mathrm{Na}-\mathrm{Cl}$ \\
\hline \multirow[t]{2}{*}{$\begin{array}{l}\text { Artesian Water in the } \\
\text { Crystalline Terrain } \\
\text { (Non-thermal) }\end{array}$} & $\begin{array}{l}\text { (a). Southern } \\
\text { Lowlands }\end{array}$ & $\begin{array}{l}\text { South West to } \\
\text { Southern } \\
\text { Highlands }\end{array}$ & $\begin{array}{l}\text { Non-mixed, non-evaporated, } \\
\text { fast replenished }\end{array}$ & $\sim 17$ to 23 & $\mathrm{Ca}-\mathrm{HCO}_{3}$ \\
\hline & $\begin{array}{l}\text { (b). Eastern North } \\
\text { Central Lowlands }\end{array}$ & $\begin{array}{l}\text { North Eastern } \\
\text { Highlands }\end{array}$ & Evaporated, fast replenished & $\sim 12$ & $\mathrm{Ca}-\mathrm{HCO}_{3}$ \\
\hline \multirow[t]{5}{*}{$\begin{array}{l}\text { Artesian Water in the } \\
\text { Crystalline Terrain } \\
\text { (Thermal) }\end{array}$} & (a). Mahapellessa & Lowlands & $\begin{array}{l}\text { Isotopically slightly enriched, } \\
\text { high mineralized and deeply } \\
\text { percolated }\end{array}$ & $>50$ & $\mathrm{Na}-\mathrm{Cl}$ \\
\hline & $\begin{array}{l}\text { (b). Wahawa Artesian } \\
\text { Well }\end{array}$ & Highlands & Intensely rock water interacted & $>50$ & $\mathrm{Na}-\mathrm{SO}_{4}$ \\
\hline & $\begin{array}{l}\text { (c). Nelumwewa and } \\
\text { Wahawa }\end{array}$ & $\begin{array}{l}\text { Intermediate } \\
\text { Zone }\end{array}$ & $\begin{array}{l}\text { Significantly mixed with } \\
\text { recently precipitated and } \\
\text { evaporated water, deeply } \\
\text { percolated }\end{array}$ & $\sim 10-13$ & $\mathrm{Na}-\mathrm{SO}_{4}$ \\
\hline & (d). Mahaoya & $\begin{array}{l}\text { Intermediate } \\
\text { Zone }\end{array}$ & $\begin{array}{l}\text { Evaporated, rock water } \\
\text { interacted, deeply percolated }\end{array}$ & $>50$ & $\mathrm{Na}-\mathrm{SO}_{4}$ \\
\hline & $\begin{array}{l}\text { (e). Kanniyai } \\
\text { (f) Rankihiriya }\end{array}$ & $\begin{array}{l}\text { Highlands } \\
\text { Intermediate } \\
\text { Zone }\end{array}$ & $\begin{array}{l}\text { Non-mixed, non-evaporated, } \\
\text { lower mineralized, deeply } \\
\text { percolated (Probably through } \\
\text { quartzite) }\end{array}$ & $>50$ & $\mathrm{Ca}-\mathrm{HCO}_{3}$ \\
\hline \multicolumn{2}{|c|}{$\begin{array}{l}\text { Spring Water in the Crystalline Terrain } \\
\text { (Non-thermal) }\end{array}$} & Lowlands & $\begin{array}{l}\text { Recently precipitated, shallow } \\
\text { percolated }\end{array}$ & $\sim 17$ to 23 & $\mathrm{Ca}-\mathrm{HCO}_{3}$ \\
\hline
\end{tabular}

Abbreviations: MRT, Mean residence time 